\title{
A-I (C3HC4-type)
}

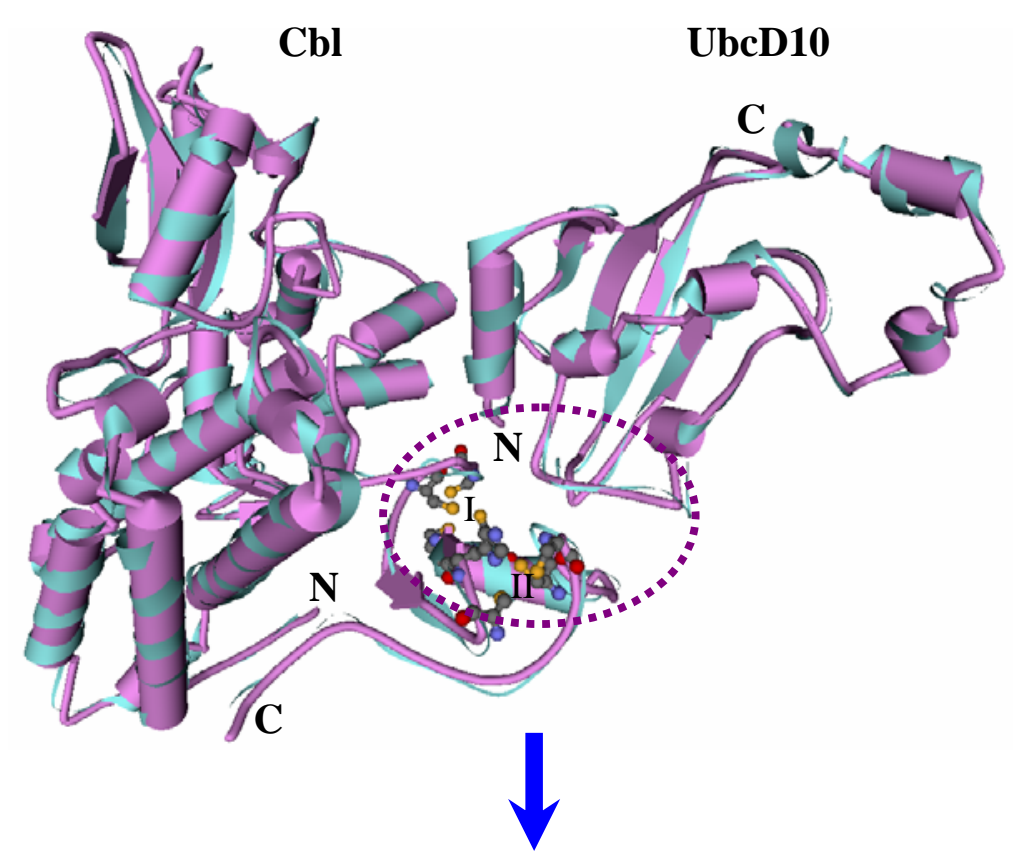

A-II

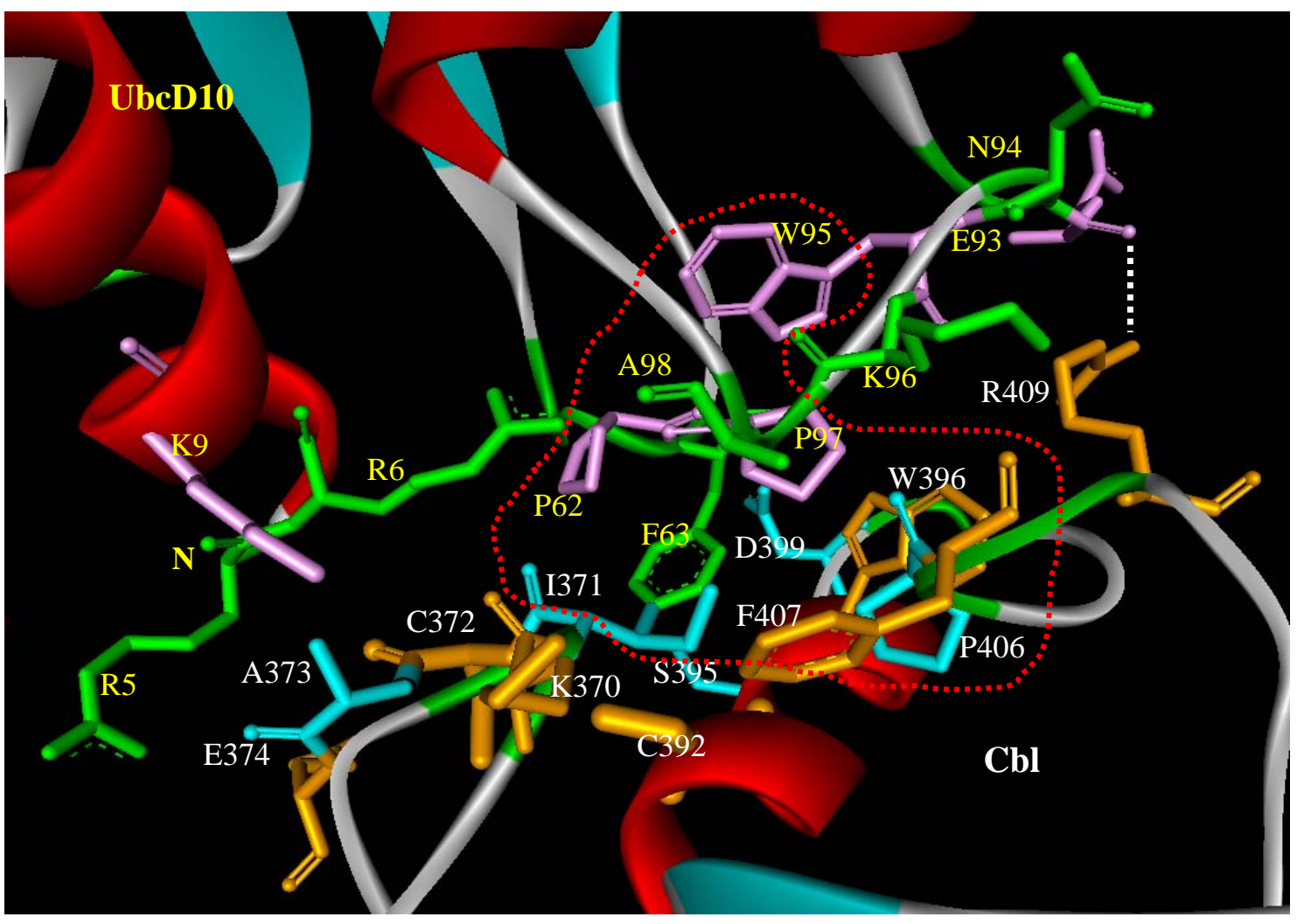


B-I (C3HC4-type)
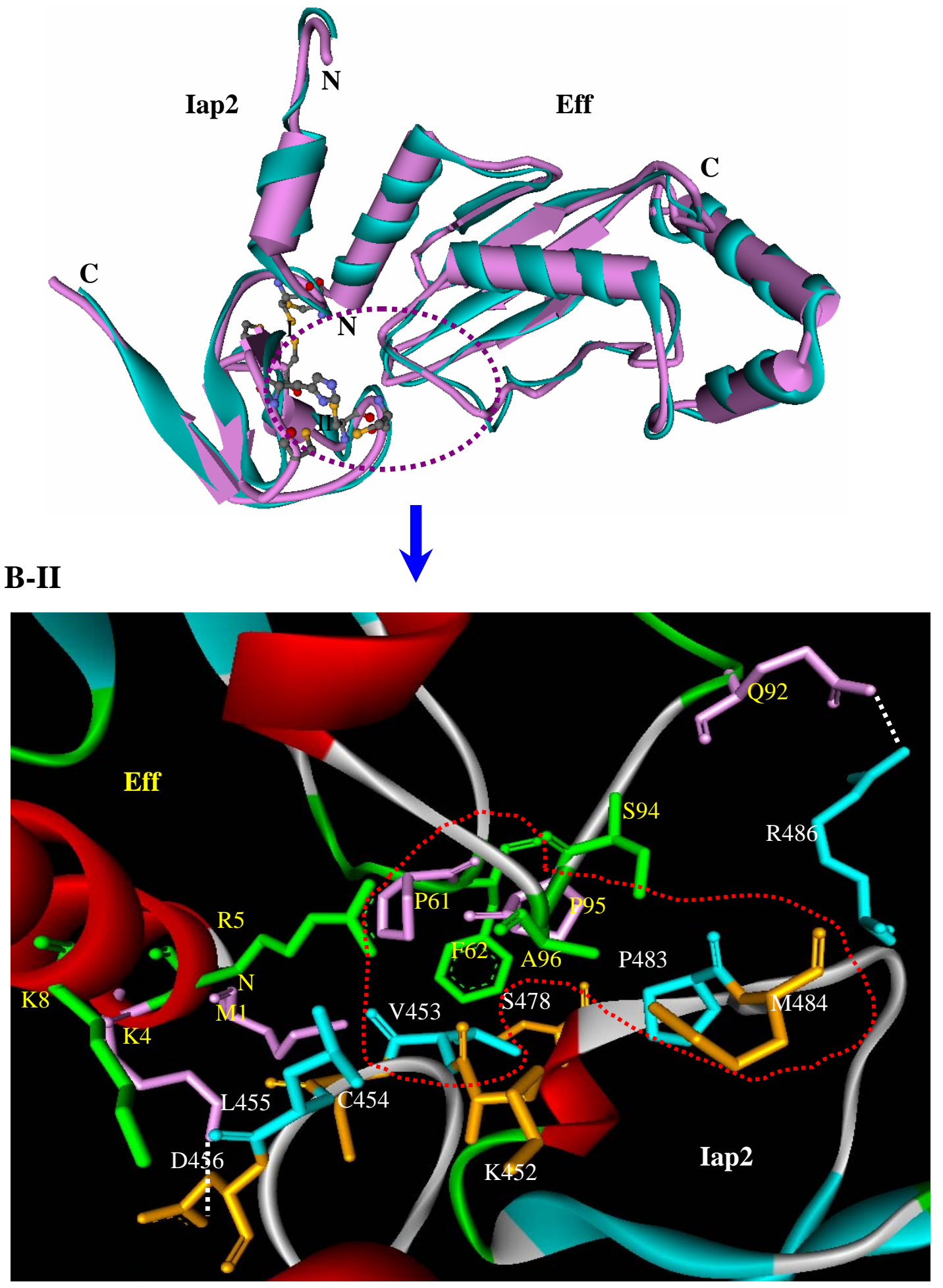
C-I (C3H2C3-type)

C-II
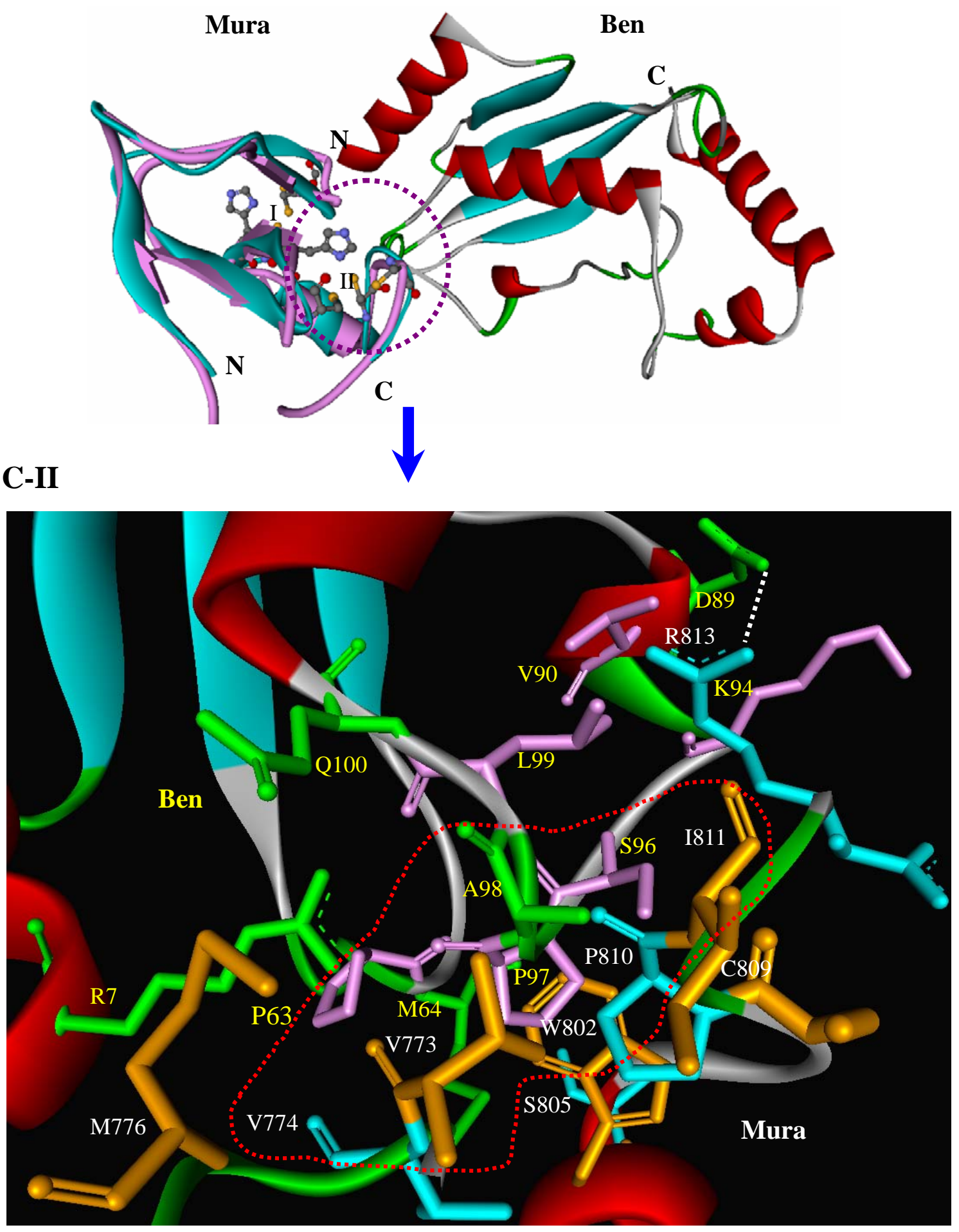

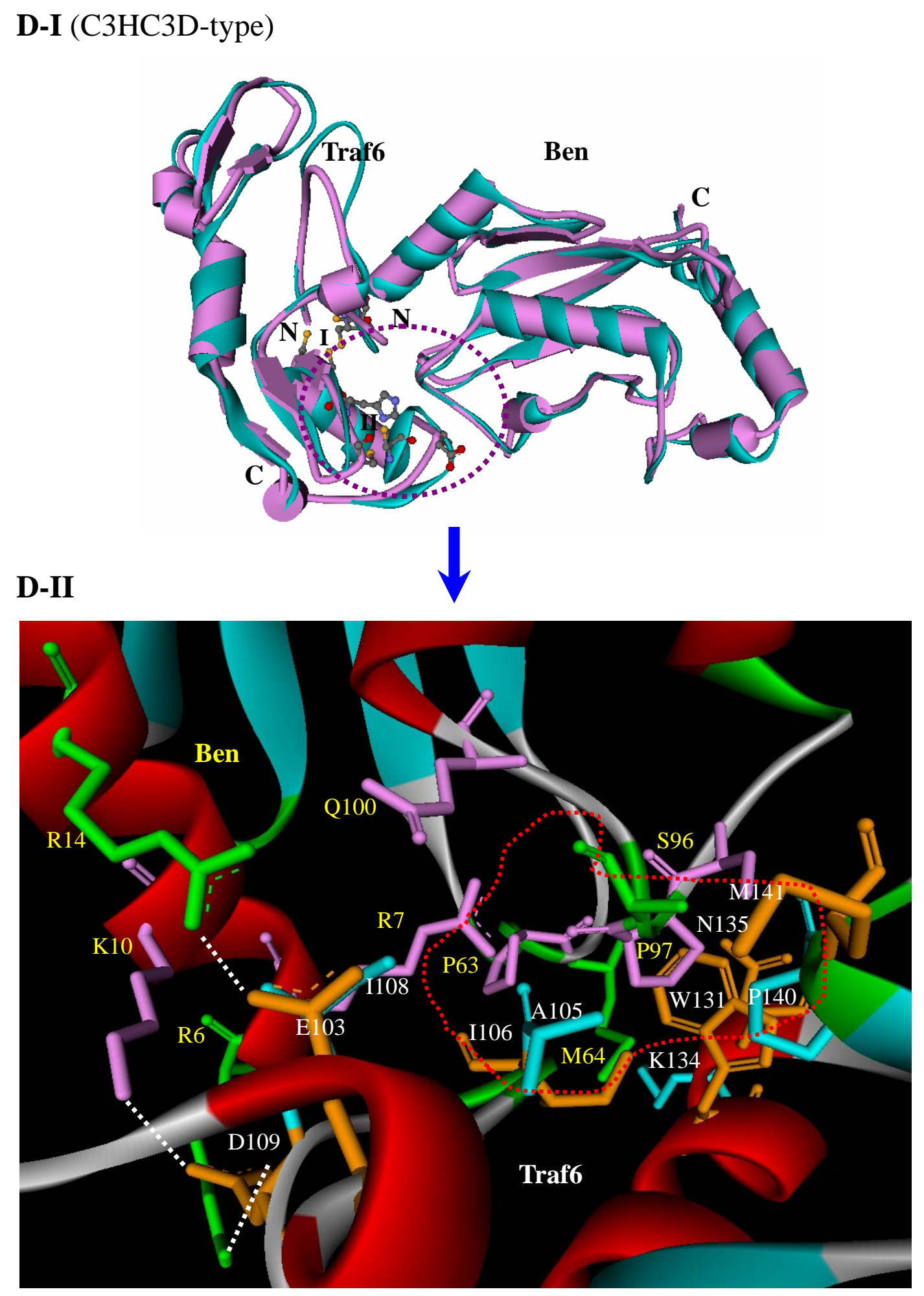


\section{E-I (C4C4-type)}

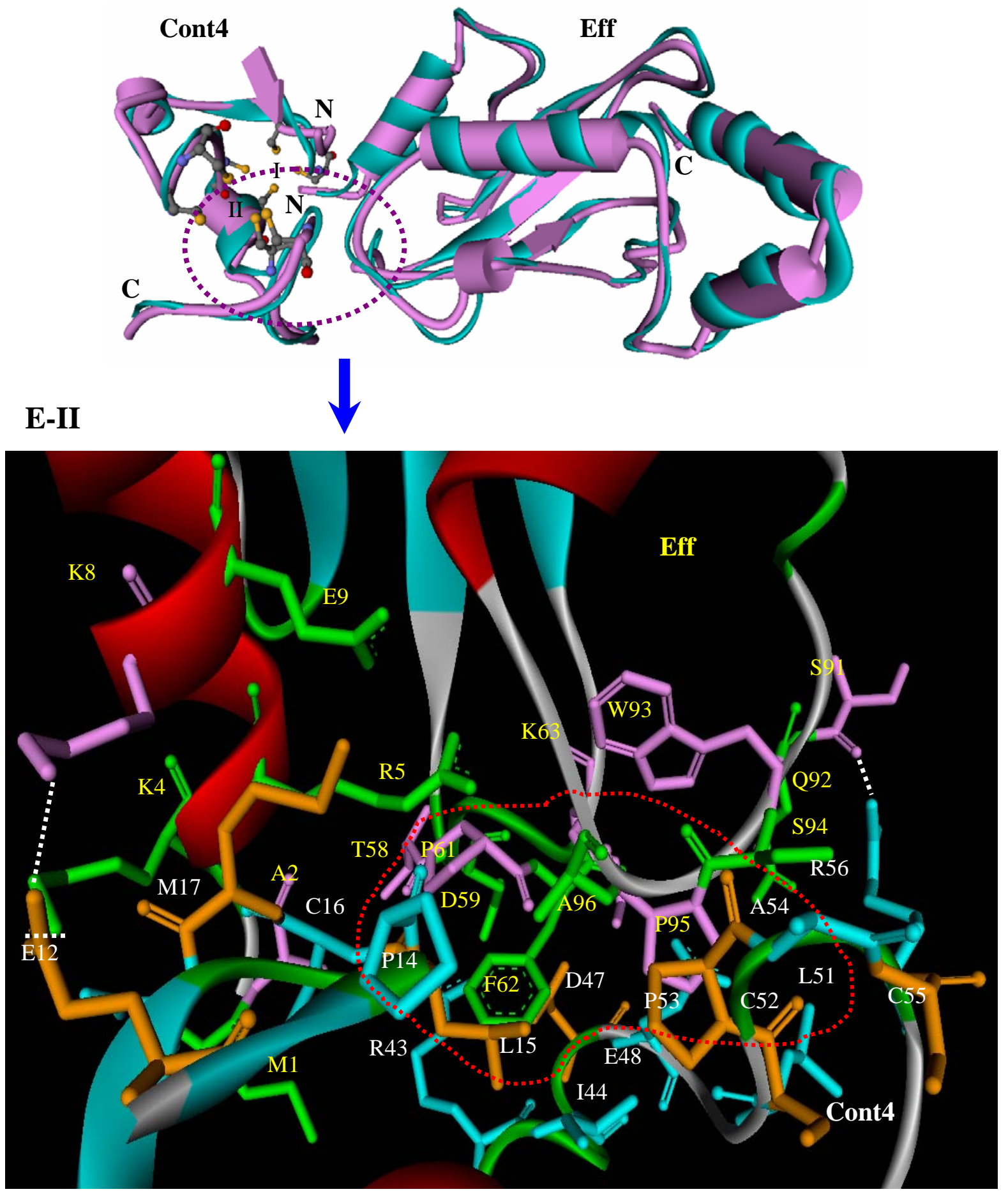




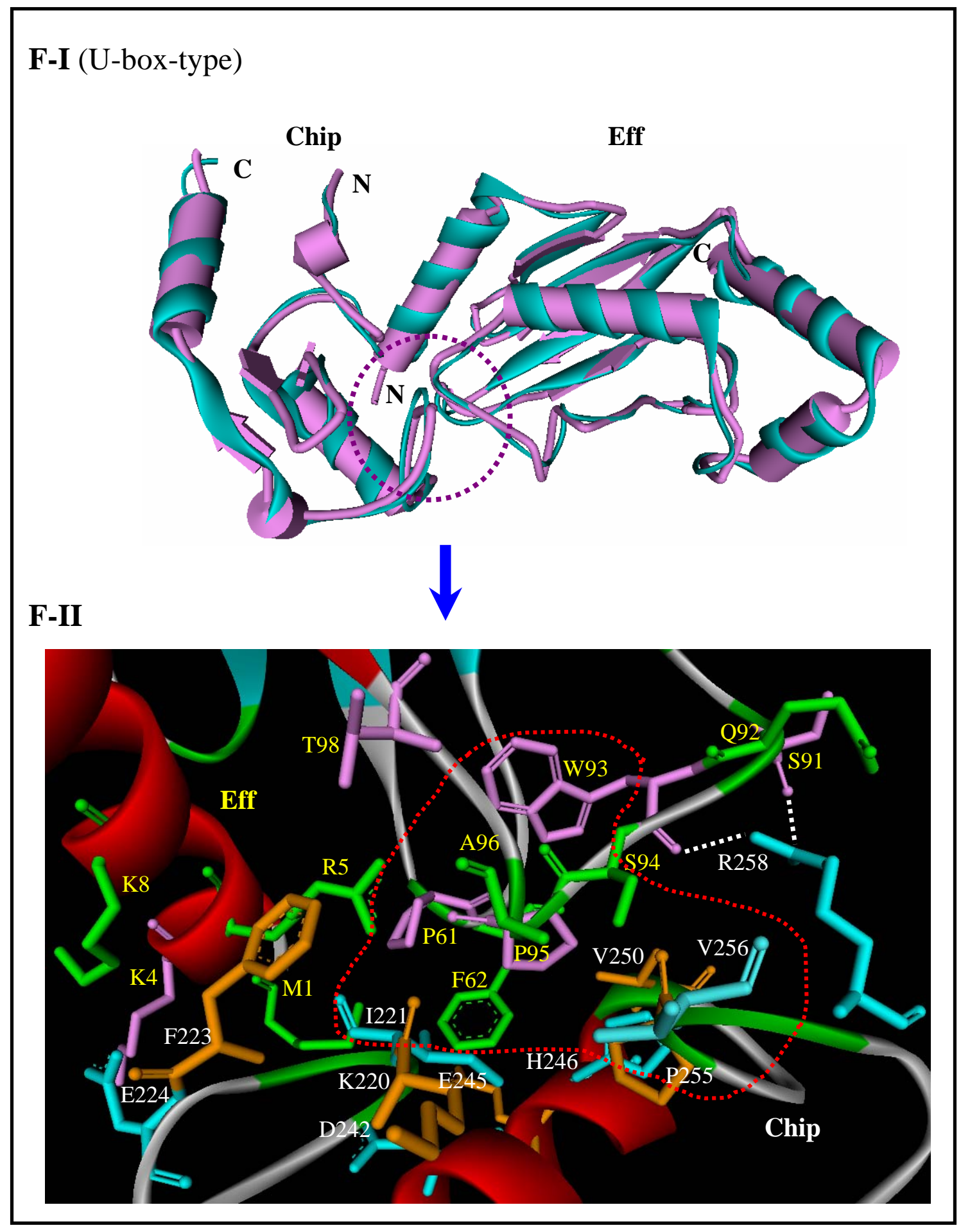


G-I (U-box-type)
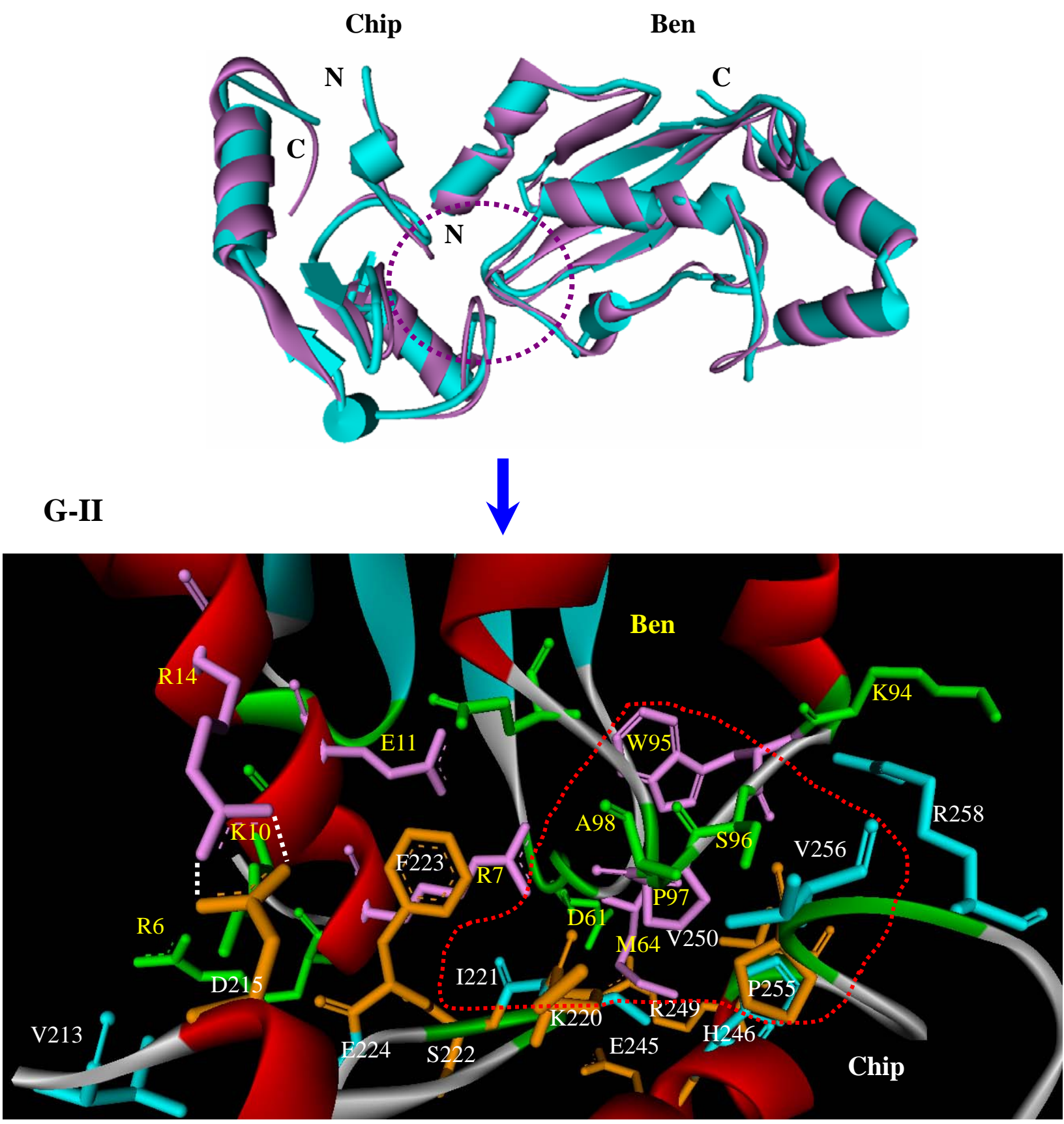

Figure S7. Cartoons of 3Ds complexes of RING-E3 and E2 pairs. A: Cbl-UbcD10; B: Iap2-Eff; C: Mura-Ben; D: Traf6-Eff; E: Cont4-Eff; F: Chip-Eff; G: Chip-Ben. A-I to G-I: Targets and templates were respectively represented by the purple ribbon models and the light blue schematic models. Structural similarity between them was shown by superimposition of their structures (N, amino terminal; C, carboxyl terminal). Cantact interfaces of RING-E3 and E2 complexes were illuminated by 
purple dot circles. I and II represent the first and the second Zn-binding sites. The two sites are respectively located on two sides of RING-E3 domain, by which form a characteristic “cross-brace” Zinc-binding topology of RING domains. A-II to G-II: Close-up view of interaction residues in the intermolecular interfaces of their 3Ds complexes. The side chains of E2 and RING-E3 3Ds complexes involved in E2/E3 interaction were presented by solid ribbon. Resides that make significant directly contacts observed in the modeling complexes were presented by stick model, and were numbered by precursor peptides. The numbers for all resides in the figure correspond to those in the text and the tables. The interaction residues in the interface were respectively indicated by yellow (E2s) and white (RING-E3s) letters. The Conserved hydrophobic contacts of intermolecular interfaces observed in the modeling complexes were highlighted by red dot circles. Hydrogen bonds of intermolecular interfaces of complexes formed by carbonyl-group oxygen and amino-group hydrogen were showed by white dot lines. 